\title{
The Future of Psychiatry
}

\author{
Suneeta Kumari* and Paras Chand \\ Howard University Hospital, Saint Luke's University Hospital, USA \\ Received: 制June 27, 2018; Published: 制 September 10, 2018 \\ *Corresponding author: Suneeta Kumari, Project Manager, Howard University Hospital, Washington DC, USA
}

\section{Mini Review}

A mental disorder is characterized as an illness that influences an individual's mood, alters psychological management and causes cognitive disposition. In the U.S., 1 in 5 Americans experiences a mental illness in a given year [1]. Furthermore, nearly 1 in 25 adults in America inhabit and live with a severe mental disorder through a given year [2]. According to NAMI (National Alliance on Mental Illnesses), schizophrenia, bipolar disorder, major depression and anxiety disorders have become increasingly prevalent throughout the U.S [1]. As a result, depression has become the leading cause of disability worldwide through the indication of multiple risk factors, such as poor diet, smoking, poor sleep hygiene, low vitamin D levels and changes in gut permeability. These risk factors present deliberate complications and may derive chronic conditions; for instance, hypertension, diabetes, arthritis, lung disease (COPD) and congestive heart failure arrogate $80 \%$ of total costs for all chronic conditions. Moreover, disorders relating to neuropsychiatric notions are the top causes of disability in the U.S [3].

This dilemma provides factors to express the importance of mental health and psychiatry as a critical component to many lives across the world. The practice of psychiatry is not only increasingly changing its demographics through scientific and technological processes, but it also presents new growth of diversity and innovation in our society. Patient expectations are changing, not in psychiatry solely, but medicine as a whole. Therefore, transforming the position of this environment is crucial for the modifications and advancements in medicine in the quickly revolving sphere of healthcare. For instance; Google and other technology companies have become affiliated with the National Institute of Mental Health to collaborate on their life sciences division, which demonstrates their commitment for a transformational impact on the methods of composing and delivering healthcare. The landscape of psychiatry, specifically new mental health technology is changing, such as the spheres of provider platforms (i.e. Valera Health and Ginger. io). Valera Health is a healthcare organization that utilizes mobile technology in order to help patients and their care teams connect when assistance is needed.

This allows patients to communicate to healthcare professionals immediately and whenever an urgent issue arises. Gingerio is also another cooperative healthcare provider, as they offer online, emotional support to a patient's personalized diagnosis. This resolves patient barriers, creating a support system to those who require it at any time of the day. These platforms continue to become progressively favored by psychiatrists, as they exploit the benefits of utilizing contemporary methods. In addition to the previous advancements in the field of psychiatry, asynchronous telepsychiatry acts as a radiologist assessing sets of scans through reviewing computer analysis interviews. The application of telepsychiatry provides patient care to individuals otherwise difficult to reach. The process and purpose of telemedicine is not to replace local mental health resources, rather to enhance current capabilities and serve vast majority of patient population. Tele psychiatric patients are implemented into regular clinic schedules, as they collaborate with reliable rural clinic. The concept includes attaining a more professional access to information about patients and provides more primary-care physicians the access to psychiatric consultations [4].

The White House BRAIN Initiative (Brain Research Advancing Innovative Neurotechnology), an initiative set by the National Institute of Health that discusses future implications of neuroscience by placing emphasis on understanding the complexity of the brain through its distinct translation of thoughts, behavior and perception [5]. The BRAIN Initiative has contributed $\$ 300 \mathrm{M}$ through public and private investments in order to display its support in the research of new methods to prevent and treat disorders of the brain, such as autism, epilepsy, traumatic brain injury and schizophrenia. The number of child psychiatrists greatly precedes the quantity of children who need psychiatric help in many areas of the U.S.; therefore, the situation becomes difficult, as humans alone, are not enough to deliver healthcare effectively across a wide range of patients, each with complex and dissimilar illnesses. Moreover, in order to confront these complications, the technological aspect regarding the future of psychiatry consists of brain stimulation, neuroimaging, neurogenetics, informatics in digital and mental health and neuroinflammation.

The new emerging technology will allow psychiatrists to intervene early and substantially produce greater and more effective impacts. For instance, eye movement tests considerably identify evidence of schizophrenia and autism, though which new 
technologies allow specialists to diagnose and treat spectrum disorders and severe mental illnesses by utilizing eye tracking solutions. This emphasizes the significance of constructing a future in psychiatry by implementing new methods of technological advances in consideration of expanding the field.

Additionally, mental specialists also may have the ability to predict the chances of suicide. According to the AFSP (American Foundation for Suicide Prevention), this condition is currently the 10th leading cause of death in the U.S., whereas approximately 44, 965 Americans die of suicide each year. By applying blood gene expression biomarkers and conducting an interview, the predication of people who have suicidal thoughts is able to be determined through immune and inflammatory indications [6].

By taking advantage of neuroinformatic genetics research, an individual is able to understand that though mental disorders, such as ADHD, autism, bipolar disorder, schizophrenia and major depressive disorder may produce dissimilar symptoms in a person mentally and physically, they may also share much of their genetic underpinnings, which expresses the connection between them. Another examination that psychiatrists exploit is a speech pattern test in order to determine and detect a specific type of mental disorder by promoting computer algorithms. Through this method, specialists have the capabilities to anticipate how people may be exposed to a certain disorder, such as schizophrenia in a high exposure area. Contemporary mechanical and specialized promotion in the region of psychiatry provides, statistically, an improved quality of life for the population and the attention of growth becomes momentous for future research and study of mental disorders. A notion that portrays this analysis is the application of neuroimaging, which identifies the stage of a mental disorder through its impact on the brain of an individual [3]. By applying modern neuroimaging techniques, the brain's form and function, regarding the internal and external integrity are revealed. For instance, the application of 7-tesla MRI scanners allows an individual to view the brain as not only a system, but also at nearly microscopic scales [7].

This method of neuroimaging contains an extremely highresolution imaging sensor, whereas it may identify areas of epileptogenic focus, which are typically too minuscule to locate on an MRI and enable a detailed and accurate surgical removal of effected brain areas. Applied neuroimaging integrates structural and functional mapping of the nervous system which guides patient management to improve patient care. The field of psychiatry is rapidly developing, as new innovations and treatments become available to a population. For instance, digital therapists, or avatars (i.e. MultiSense and SimSensi) have the ability to view an individual through analyzing speech patterns and body movements in order to emotionally have a connection [2]. This is crucially significant for people who a mental disorder condition, such as autism. Autism is a severe mental disorder that effects and debilitates an individual's ability to communicate and interact effectively. According to the Centers of Disease Control and Prevention (CDC), 1 in 68 children are identified with having the condition, as the diagnosis of this mental disorder begins to increase yearly.

With the development of the Brain Power System, a wearable, smart glass technology for autism that creates a positive and relaxed mental state of the human mind, allowing them to learn cognitive and social skills. The growth of psychiatry may also present the first digital medications in the field of medicine, such as antipsychotics that have built-in sensors, whereas their purpose serves to report the body's schematics and other relevant information [8]. The system operates through reporting data from the pill's sensor to a wearable patch, which tracks ingestion of the medication. The product is generally practical for the treatment of schizophrenia and acute treatments associated with bipolar disorder. The pill generates an electrical signal when exposed to stomach acid, as it contains traces of copper, magnesium and silicon. Through the promotion and evolution of psychiatric progress, the future of this area of medicine may expand brain networks or overpower motor and cognitive issues by the application of extending the opportunities of today's capabilities.

\section{Conclusion}

The landscape of psychiatry is rapidly changing as new and developing options become available to patients. The study of the field has become increasingly crucial throughout recent years and the future not only offers success, it demonstrates enrichment to all patients.

\section{References}

1. Mental Illness.

2. (2016) Autism Spectrum Disorder (ASD)

3. Eierud C, Craddock RC, Fletcher S, Aulakh M, King Casas B, et al. (2014) Neuroimaging after mild traumatic brain injury: review and metaanalysis.

4. Deslich S, Stec B, Tomblin S, Coustasse A (2013) elepsychiatry in the 21st Century: Transforming Healthcare with Technology.

5. Jorgenson LA, Newsome W T, Anderson D J, Bargmann CI, Brown EN, Deisseroth K, Wingfield JC (2015) The BRAIN Initiative: developing technology to catalyze neuroscience discovery.

6. Lopez JP, Kos A, Turecki G (2018) Major depression and its treatment: microRNAs as peripheral biomarkers of diagnosis and treatment response.

7. Reid MA, Salibi N, White DM, GawneTJ, Denney TS, Lahti AC (2018) 7T Proton Magnetic Resonance Spectroscopy of the Anterior Cingulate Cortex in First-Episode Schizophrenia.

8. Yoshimura B, Sakamoto S, Sato K, Takaki M, Yamada N (2017) Predictors of remission during acute treatment of first-episode schizophrenia patients involuntarily hospitalized and treated with algorithm-based pharmacotherapy: Secondary analysis of an observational study. 
ISSN: 2574-1241

DOI: 10.26717/BJSTR.2018.08.001713

Suneeta Kumari. Biomed J Sci \& Tech Res

(c) (9) This work is licensed under Creative Submission Link: https://biomedres.us/submit-manuscript.php

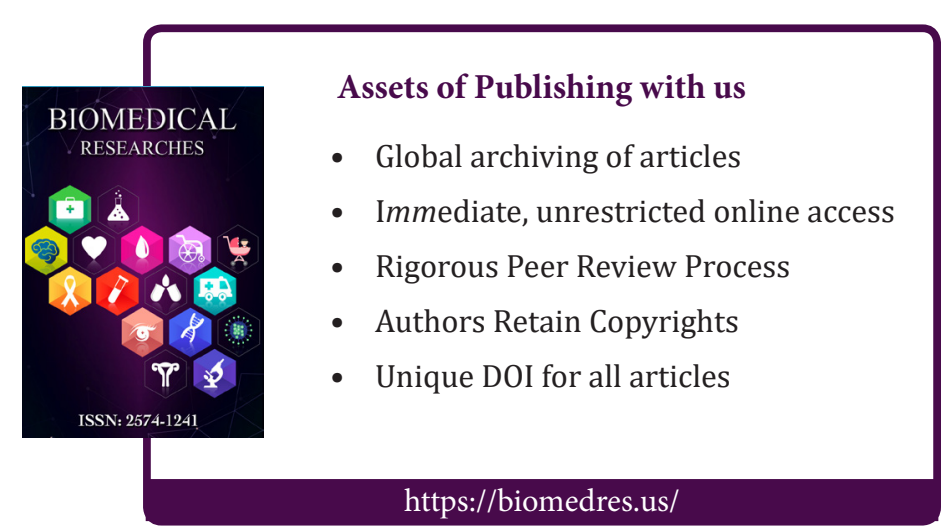

